\title{
Gustatory and olfactory dysfunction in older adults: a national probability study*
}

\author{
Sanne Boesveldt ${ }^{1,2}$, Stacy Tessler Lindau ${ }^{3}$, Martha K. McClintock ${ }^{4}$, Thomas \\ Hummel $^{5}$, Johan N. Lundström ${ }^{1,6,7}$ \\ 1 Monell Chemical Senses Center, Philadelphia, PA, USA \\ 2 Division of Human Nutrition, Wageningen University, Wageningen, the Netherlands \\ 3 Department of Obstetrics and Gynecology and Medicine-Geriatrics and Chicago Core on Biomeasures in \\ Population-Based Aging Research at the NORC and University of Chicago Center on Demography and \\ Economics of Aging, University of Chicago, Chicago, IL, USA \\ 4 Department of Human Development, University of Chicago, Chicago, IL, USA \\ 5 Smell \& Taste Clinic, Department of Otorhinolaryngology, University of Dresden Medical School, Dresden, \\ Germany \\ 6 Department of Psychology, University of Pennsylvania, Philadelphia, PA, USA \\ 7 Department of Clinical Neuroscience, Karolinska Institute, Stockholm, Sweden
}

Background: Olfactory and gustatory functions have not been well characterized in older adults in the US. Consequently, their relationships to sociodemographic characteristics, as well as physical and mental health, were studied in a large national probability sample $(n=$ 3005 ) using brief validated tests of chemosensory function.

Methods: A five-odour identification test and taste-impregnated strips of filter paper (sweet, sour, bitter, and salty) assessed the ability to identify chemosensory stimuli.

Results: Severe gustatory dysfunction (14.8\%) was more prevalent than severe olfactory dysfunction $(2.7 \%)$. Age, education and sex were independently associated with performance on both the olfactory and gustatory identification tasks. Higher scores were associated with female sex, higher level of education, and lower age. Odour identification scores exhibited a positive, albeit weak, correlation with BMI, and food-related odours were better identified than non-food odours. In addition, odour identification performance was also negatively associated with depressive symptoms.

Conclusions: These data demonstrate a high prevalence of severe gustatory and, to a somewhat lesser extent, olfactory dysfunction in a population-based sample and demonstrate that even brief tests are capable of detecting correlations between both chemical senses and relevant health measures outside a clinical setting.

Key words: gustation, olfaction, age, prevalence, sex

\section{INTRODUCTION}

Older adults comprise the fastest growing segment of the population. In the US alone, approximately 68.5 million people are currently older than 55 years of age, a figure estimated to surpass 108 million ( $29 \%$ of the US population) by the year 2030. A significant number of those will experience age-related sensory loss ${ }^{(1-4)}$ that will potentially impair overall health and well-being. Chemosensory function (olfaction and gustation) plays an important role in the safety and quality of the life of older adults. Individuals with compromised chemosensory function are at greater risk for food poisoning and cooking or heating gas injuries due to their inability to identify spoiled food or detect the odour warning of a gas leak. Loss of chemosensory function has also been closely linked to inadequate nutritional intake, reduced social pleasure, aging anorexia, and other related diseases ${ }^{(5-7)}$. Moreover, since olfactory dysfunction has been identified as an early marker of neurodegenerative disorders ${ }^{(8-11)}$, assessment of olfactory function may become an important element of early diagnostic strategies for the elderly. Chemosensory function has not only been linked to physical health, but also to psychological well-being; olfactory dysfunction has also been associated with both mood and affective disorders in younger and older adults ${ }^{(12,13)}$.

Although several large studies report the prevalence of olfactory dysfunction in the general population, few population-based studies have included a substantial older adult population. The Epidemiology of Hearing Loss Study, a population-based study of sensory loss and aging in older adults, demonstrated that the prevalence of objective olfactory impairment (cor- 
rectly identifying fewer than 6 out of 8 odorants) among these subjects is remarkably high (24.5\%) and grows more prevalent with age, to $62.5 \%$ in 80 to 97 -year-olds. In contrast, selfreported olfactory loss was substantially lower $(9.5 \%)$ and therefore not a sensitive indicator of objective olfactory impairment ${ }^{(14)}$. The National Geographic Smell survey collected data from a self-administered 6-item odour identification test from 1.2 million readers with a wide age range ${ }^{(15)}$. Based on these data, an estimated $1 \%$ of the participants demonstrated a severe olfactory dysfunction. In other words, the prevalence of olfactory dysfunctions in the general population is not clear.

So far, there is only one study that investigated the prevalence of gustatory dysfunction in a non-clinical population (16): the Dortmund Health Study, a cross-sectional population survey of adults aged 25 - 75 years, showed that approximately $20 \%$ exhibited some gustatory dysfunction and recognized three or fewer out of four tastes presented by a spray on the tongue. The combined occurrence of smell and taste dysfunction in that study was observed in $6.3 \%$ of participants.

In general, community-based studies of interactions between chemosensory function and mental or physical health in an aged population, despite evidence supporting a close link, are underrepresented in the literature. The National Social Life, Health and Aging Project (NSHAP) was designed to explore chemosensory function in relation to physical, psychological, and cognitive dimensions of health in a national probability sample of 3,005 community-residing older adults. Brief olfactory and gustatory tests ${ }^{(17,18)}$ were used to estimate the prevalence of severe olfactory or gustatory dysfunction, to explore the relationship of chemosensory function with physical, psychological and cognitive dimensions of health, and to explore whether sex differences in chemosensory function observed in young adults are also found among older adults.

\section{METHODS}

\section{Participants}

This study is part of the National Social Life, Health and Aging Project (NSHAP), a population-based study of community-dwelling older adults in the greater Chicago area (IL, USA). Details of the study design have been previously described ${ }^{(19)}$. In the first wave of the NSHAP, data were collected from a nationally-representative probability sample of 3005 American adults ranging from 57 to 85 years of age (mean age 69.3 years, $\mathrm{SD}=7.9 ; 1455$ men, 1550 women). Home-based biomeasure collection and face-to-face interviews were performed by trained field staff and included objective and participative measures of olfaction, gustation, vision, tactile sensation and a questionnaire-based measure of auditory function ${ }^{(20)}$. The protocol was approved by the institutional review boards of the University of Chicago and NORC (National Opinion Research Center); all respondents provided written informed consent.

\section{Gustatory function testing}

Assessment of gustatory function was performed using a series of taste-impregnated strips of filter paper ${ }^{(17,21)}$. Four strips were presented once to each participant in the same order; the first tasted sour $(0.165 \mathrm{~g} / \mathrm{ml}$ citric acid $)$, the second bitter (0.0024 $\mathrm{g} / \mathrm{ml}$ quinine-hydrochloride), the third sweet $(0.2 \mathrm{~g} / \mathrm{ml}$ sucrose), and the fourth salty ( $0.1 \mathrm{~g} / \mathrm{ml}$ sodium chloride). These concentrations correspond to the second highest concentrations (and $10^{\text {th }}$ percentile) of the validated 'taste strips' (17, 21). Before and during presentation of each strip, four descriptors, 'salty', 'sweet', 'bitter', and 'sour', were provided orally and written on a computer screen and/or paper. Participants were asked to take a sip of water before tasting each strip and were then instructed to place the strip on the center of their tongue and identify the tastant using one of the four descriptors available, without the option to change their response later.

\section{Olfactory function testing}

Olfactory function was assessed using a validated brief odour identification test ${ }^{(18)}$. Five odorants in suprathreshold concentration were administered using commercially-available felt-tip pens. The odorants were presented in a multiple forced-choice format, each with four descriptors that were given verbally and written on a computer screen and/or paper before and during each odour presentation. The response set was as follows (in order of administration, with the target odorant indicated in italics): 1) chamomile, raspberry, rose, cherry; 2) smoke, glue, leather, grass; 3) orange, blueberry, strawberry, onion; 4) bread, fish, cheese, ham; 5) chive, peppermint, pine, or onion. Following a forced-choice paradigm respondents were not permitted to answer 'don't know.' Each pen was held approximately $2 \mathrm{~cm}$ in front of the nostrils for 2 - 3 seconds, with an interval of $20-30$ seconds between each pen.

Participants were given a choice of recording their answers to the olfactory and gustatory assessments directly on the computer or on a sheet of paper. Sixty-one percent of participants in the olfaction protocol chose the paper version, while $57 \%$ of participants in the taste protocol chose the paper version.

\section{Data analysis}

Gustatory and olfactory scores were defined as the number of correct responses (taste ID, odour ID). Participants with missing values on the tests were excluded from analysis; for gustatory function testing, $1.0 \%$ of participants refused to answer, $1.3 \%$ of participants did not know the answer, $8.1 \%$ of participants did not provide an answer, $1.1 \%$ of responses were missing in error, and $8.0 \%$ were not applicable; for olfactory function testing, $5.5 \%$ of participants refused to answer, $0.1 \%$ of responses were missing in error, and $1.9 \%$ were not applicable. The numbers of individuals included in the analysis are detailed in each section below for clarity.

In a previous study, none of normosmic participants scored 0 or 1 on the brief olfactory test ${ }^{(18)}$. We therefore classified participants as having severe olfactory dysfunction if they scored $\leq 1$, indicating less than $25 \%$ accuracy (or 'chance', since the test used a multiple (4)-choice design). For consistency, we used the same cut-off to classify severe gustatory dysfunction. Olfactory identification was separated into 'food' (peppermint, 
fish, orange) and 'non-food' (rose, leather) items. For further analysis, participants with known medical causes for ageusia or anosmia (e.g. major head trauma $\mathrm{n}=131$, Alzheimer's disease $n=26$, nasal surgery $n=214$, current cold $n=17$, current allergies $n=6$, chronic sinusitis $n=4$, other $n=7$ ) were excluded.

Multiple linear regression analysis was used to explore the respective contributions of the following variables of interest to olfactory and gustatory test scores: 1) sociodemographic characteristics including age, sex, and education (less than high school, high school diploma/equivalency, associate's [2-year college] or post-high school vocational certificate, bachelor's [4-year college] degree or more); 2) physical health measures including body mass index $\left(\mathrm{BMI}\right.$, in $\left.\mathrm{kg} / \mathrm{m}^{2}\right)$ and medication use (number of different medications taken regularly, including prescription and non-prescription medications, over-the-counter medicines, vitamins, and herbal and alternative medicines); and 3) mental health, including depressive symptoms (measured by an 11-item version of the Center for Epidemiologic Studies Depression Scale [CES-D] ${ }^{(22,23)}$, where higher scores reflect increased dysphoria).

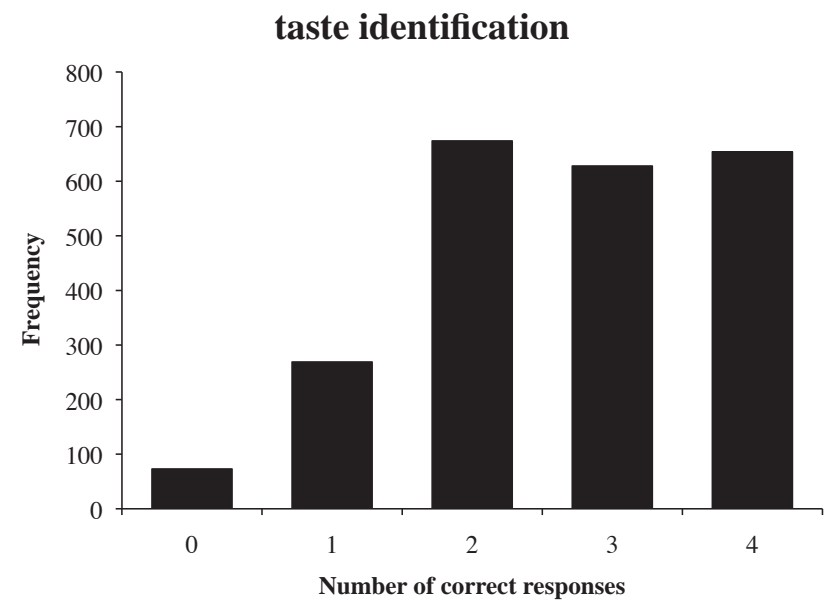

Figure 1a. Frequency distribution of number of correct responses to the gustatory identification test.

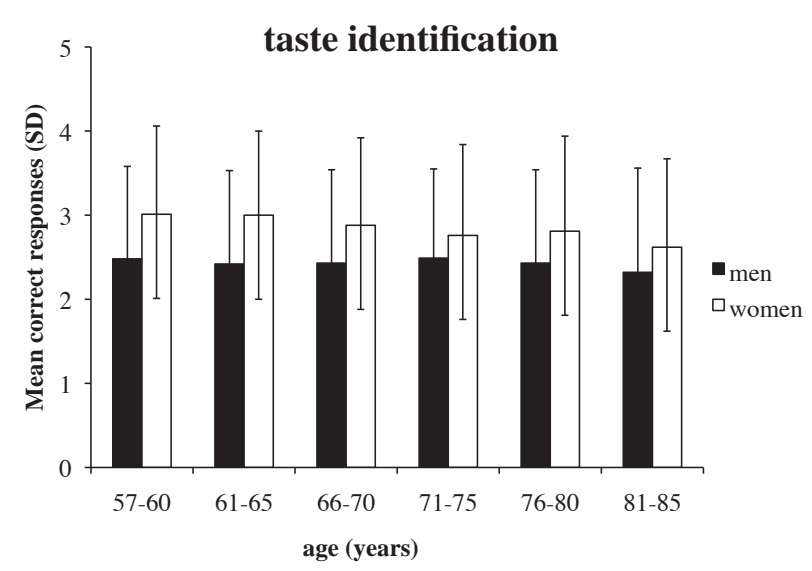

Figure 2a. Mean gustatory identification score, and standard deviations, plotted per age group and sex.
Bivariate Pearson correlation coefficients were computed to determine the correlation between gustatory and olfactory scores and the aforementioned variables. A Student's t-test was performed to explore sex differences in gustatory and olfactory function. All analyses were performed with SPSS 17.0 Software (Chicago, IL, USA).

\section{RESULTS}

For participant characteristics, and variables, see Table 1.

\section{Gustatory performance}

The prevalence of severe gustatory dysfunction in the tested population $(\mathrm{n}=2419)$ was $14.8 \%$ (women $10.2 \%$, men $19.7 \%$; $\mathrm{t}[2148]=6.582, \mathrm{p}<0.001)$. 'Sweet' taste was identified correctly most often $(86.8 \%)$, whereas 'sour' taste was accurately identified least often (39.4\%, see Table 2). After exclusion of participants with known medical causes of ageusia (leaving $\mathrm{n}=$ 2298), mean gustatory test score was 2.7 ( $\mathrm{SD} \pm 1.1$; Figure 1a), and women performed better than men (women 2.9, men 2.4; $\mathrm{t}$ $[2251]=9.380, \mathrm{p}<0.001)$.

Multiple regression analysis revealed that sex, education and

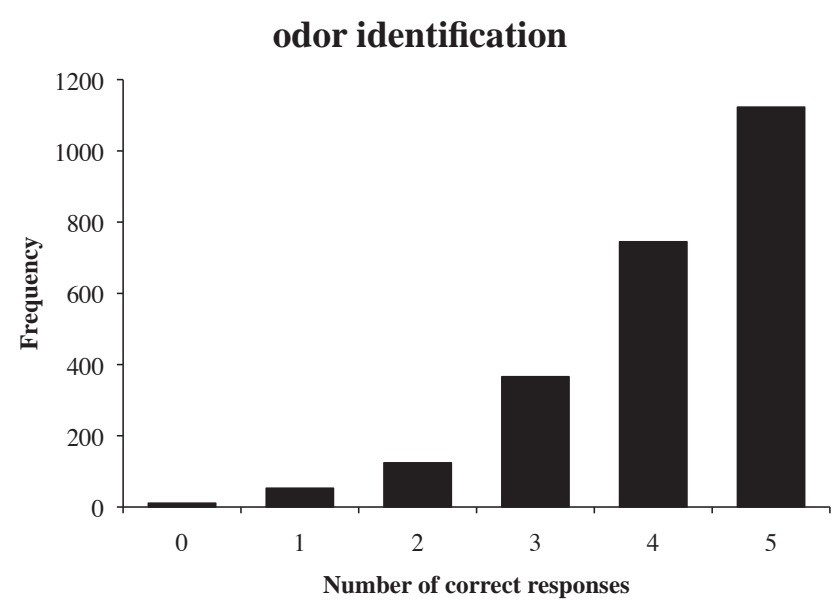

Figure 1b. Frequency distribution of number of correct responses to the olfactory identification test.

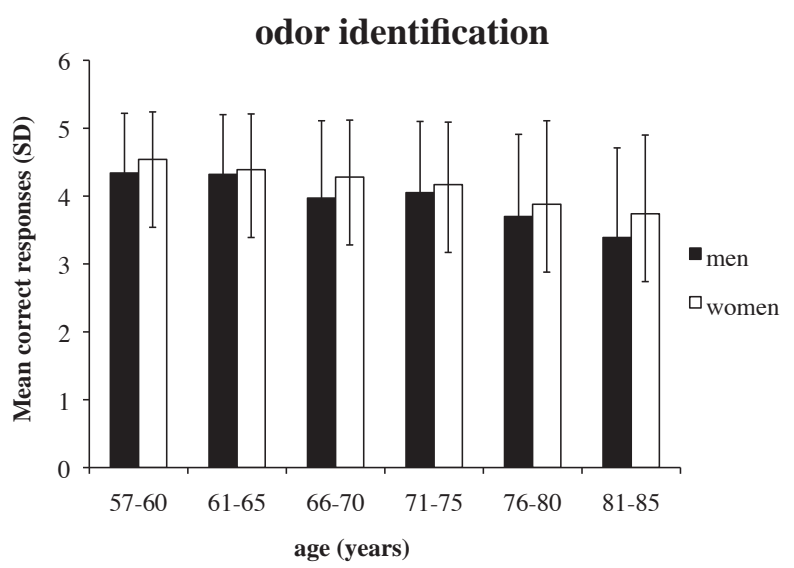

Figure 2b. Mean olfactory identification score, and standard deviations, plotted per age group and sex. 
age explained a total of $4.4 \%$ of the variance in gustatory ID score (Table 3), a smaller proportion of variance than could be explained in olfactory performance by these same factors. Higher scores were associated with female sex, higher level of education, and lower age.

Gustatory test scores were significantly correlated with age (both sexes, $r=-0.047, p=0.024$; men $r=-0.018, p=0.558$; women $r=-0.108, p<0.001$; Figure 2a), education (both sexes, $r=0.048, p=0.023$; men, $r=0.093, p=0.002$; women, $\mathrm{r}=0.050, \mathrm{p}=0.085$ ), and number of medications taken (both sexes, $r=0.056, p=0.007$; men, $r=0.019, p=0.522$; women, $r=0.042, p=0.147)$. No correlation was found between gustatory test scores and BMI $(r=0.002, p=0.915)$ or depressive symptoms $(r=0.001, p=0.943)$.

\section{Olfactory performance}

The prevalence of severe olfactory dysfunction in the tested population $(\mathrm{n}=2,778)$ was $2.7 \%$ (women $2.2 \%$, men $3.2 \%$; $\mathrm{t}$ [2628] $=1.723, \mathrm{p}=0.085)$. Of the individual odours, 'peppermint' was accurately identified most often $(91.6 \%)$, whereas 'leather' was correctly identified least often ( $70.5 \%$, see Table 2$)$.

After exclusion of participants with known medical causes of dysfunctions (leaving $\mathrm{n}=2,422$ ), mean olfactory identification score was 4.1 ( $\mathrm{SD} \pm 1.0$; Figure $1 \mathrm{~b}$ ), and women performed better than men (women 4.2, men 4.1; $\mathrm{t}[2346]=3.633$, $\mathrm{p}<0.001$ ). Food items were accurately identified more often than nonfood items ( $88.4 \%$ vs. $73.7 \%$ ).

Multiple regression analysis revealed that age, education, sex, and depressive symptoms explained a total of $9.1 \%$ of the variance in olfactory identification score (Table 3). Higher odour identification scores were associated with lower age, higher level of education, female sex, and fewer depressive symptoms.

Olfactory test scores were significantly correlated with age, (both sexes, $r=-0.250, p<0.001$; men $r=-0.260, p<0.001$; women $r=-0.254, p<0.001$; Figure 2b), BMI (both sexes, $r=$ $0.060, \mathrm{p}=0.004$; men $\mathrm{r}=0.161, \mathrm{p}<0.001$; women $\mathrm{r}=-0.029$, $\mathrm{p}=0.321$ ), depressive symptoms (both sexes, $\mathrm{r}=-0.095, \mathrm{p}<$ 0.001 ; men $r=-0.129, p<0.001$; women $r=-0.078, p=0.006$ ), and education (both sexes, $\mathrm{r}=0.173, \mathrm{p}<0.001$; men $\mathrm{r}=0.202$, $\mathrm{p}<0.001$; women $\mathrm{r}=0.157, \mathrm{p}<0.001)$. No correlation was found between olfactory test scores and number of medications

Table 1. Participant characteristics (total population).

\begin{tabular}{l|l}
\hline Sex (m / f) & 1455 / 1550 \\
Age (range / mean / SD) & $57-85$ / 69.3 / 7.9 \\
Taste ID scores (range / mean / SD) & $0-4$ / 2.7 / 1.1 \\
Odour ID scores (range / mean / SD) & $0-5$ / 4.1 / 1.0 \\
BMI (range / mean / SD) & $14.1-75.6$ / 29.1 / 6.3 \\
CES-D score (depressive symptoms; range / & $0-32$ / 5.6 / 5.2 \\
mean / SD) & $0-20$ / 5.2 / 3.9 \\
Number of medications taken (range / mean & \\
/ SD) & 699 / 793 / 856 / 657 \\
Education (<hs / hs, equiv / vc, assoc / bchl)
\end{tabular}

ID = identification; $<$ hs = less than highschool; hs, equiv = highschool diploma/equivalency; vc, assoc = associate's (2-year college) or post-HS vocational certificate; bchl = bachelor's (4-year college) degree or more taken $(\mathrm{r}=0.001, \mathrm{p}=0.963)$.

Combined olfactory and gustatory dysfunction was found in $0.7 \%$ of the tested population (men $0.9 \%$, women $0.6 \%$ ); whereas $15.9 \%$ of participants exhibited either severe olfactory or gustatory dysfunction (men $21.0 \%$, women $11.3 \%$ ). Olfactory test scores were significantly correlated with gustatory test scores (both sexes, $\mathrm{r}=0.133, \mathrm{p}<0.001$; men, $\mathrm{r}=0.126$, $\mathrm{p}<0.001$; women, $\mathrm{r}=0.121, \mathrm{p}<0.001)$.

\section{DISCUSSION}

As a part of the National Social Life, Health and Aging Project (NSHAP), we used brief olfactory and gustatory tests to study the two main chemical senses and determine their relation to health measures in a large community sample of older adults. The prevalence of severe gustatory or olfactory dysfunction was $14.8 \%$ and $2.7 \%$, respectively. Overall performance on both the gustatory and olfactory identification tasks were significantly explained by age, education, and sex, and odour ID performance was further explained by depressive symptoms. Foodrelated odours were better identified than non-food odours.

\section{Prevalence of olfactory and gustatory dysfunction}

The prevalence of severe olfactory dysfunction in our representative sample of U.S. older adults was $2.7 \%$, slightly lower than estimates reported in previous studies (approximately 3.5 - 5\%) in European populations ${ }^{(16,24,25)}$. This discrepancy may be due in part to our exclusion of individuals with incomplete identification test data. As a result, the estimated prevalence of severe olfactory dysfunction in our study may underestimate the actual prevalence of severe olfactory dysfunction due to exclusion of participants whose failure to choose a descriptor for one or more response sets resulted from an olfactory impairment. An estimated $1 \%$ of participants of the National Geographic Smell survey demonstrated a severe olfactory dysfunction ${ }^{(15)}$; slightly lower than in the present study. However, that survey used participants from a wide age range, and a self-administered odour identification test, which could have led to an underestimation of the actual prevalence of olfactory impairment.

As previously reported ${ }^{(26)}$, this study finds that odour stimuli representing food items were better identified than non-food items. Performance on odour identification tests generally

Table 2. Percentage of correctly identified odour and taste items.

\begin{tabular}{lc|cc}
\hline Odour item & $\begin{array}{c}\text { \% correctly } \\
\text { identified }\end{array}$ & Taste item & $\begin{array}{c}\text { \% correctly } \\
\text { identified }\end{array}$ \\
\hline Rose & 75.8 & Sour & 39.4 \\
Leather & 70.5 & Bitter & 70.0 \\
Orange & 84.8 & Sweet & 86.8 \\
Fish & 87.0 & Salty & 67.4 \\
Peppermint & 91.6 & & \\
\hline
\end{tabular}


depends not only on exposure to and familiarity with the odours in question, but also on eating habits ${ }^{(27)}$, giving rise to a likely cause for the higher identification scores for the food-related odours. However, since only a limited sample of odorants (representing both food and non-food items) was used in this study, these data should be viewed as tentative and future studies should focus on this finding in more detail by presenting a wider range of odorants, and might be able to demonstrate differences in the processing of odours related to food or non-food.

Unlike olfactory tests, standardized clinical tests of taste function are rarely available to the clinician, and literature on the prevalence of gustatory dysfunction is therefore scarce. In a recent population-based study, Vennemann and colleagues ${ }^{(16)}$ found that approximately $20 \%$ of more than 1300 participants in the ages 25 - 75 (mean age 52.1) were unable to identify all four tastes correctly, although the tests were presented at suprathreshold concentrations. This value is higher than the prevalence of severe gustatory dysfunction we report here $(14.8 \%)$. Because of the limited number of items in the gustatory and olfactory identification test, we chose to be conservative in calculating the prevalence of dysfunction, by only classifying participants as having severe gustatory or olfactory dysfunction if they scored less than $25 \%$ accuracy (or 'chance'). This could explain why the prevalence of severe olfactory or gustatory dysfunction in the present study is (slightly) lower than in previous studies that obtained data in similar populations or under similar circumstances ${ }^{(14,16,24,25)}$. However, not all participants scoring $>1$ or $>1.25$ on the taste or smell test, respectively, should consequently be classified as having normal gustatory or olfactory function, but could be considered for extended testing.

Two previous studies of gustatory dysfunction in younger adults seen in a chemosensory clinic, reported a significantly lower prevalence of severe gustatory dysfunction $(0.85 \%-4 \%)$ than the current study ${ }^{(28,29)}$. Since gustatory function is known to decrease with age ${ }^{(4,30,31)}$, the discrepancy between studies could be explained by the different age groups explored.

When analyzing the individual taste qualities, 'sour' was identified correctly least often $(39.4 \%)$, consistent with previ- ous findings by Nordin and colleagues who demonstrated a pronounced age-related loss in identification for citric acid ${ }^{(32)}$. 'Sour' was also likely to be misidentified as 'salty' (43.1\%), suggesting misidentification of taste quality, rather than specific loss of sour identification among older adults. Furthermore, others have reported that sensitivity for salty and bitter is also affected by aging ${ }^{(33)}$. In contrast, as indicated by the present data, recognition of sweet seems to be stable throughout life, i.e. aging does not affect sensitivity to each taste quality equally.

Sociodemographic influences on olfactory and gustatory function Age and sex were significant predictors of both olfactory and gustatory identification performance. This is consistent with previous research showing marked age-related declines ${ }^{(1,30,34-}$ 36) and superior female performance ${ }^{(1,30,35,37)}$ in assessments of both olfactory and gustatory function.

Both odour and taste identification performance were positively associated with education level. This finding concurs with previous studies ${ }^{(30)}$ and corresponds to the notion that chemosensory identification depends to some degree on language capacity or semantic memory for odour naming and labeling ${ }^{(38)}$, skills that are closely related to level of education.

\section{Associations between the chemical senses and mental and physical health}

In addition to the above-mentioned, well-established influences, CES-D score also accounted for a significant part of the variance in odour identification performance. Previous reports of the relationship between olfactory function and depression or depressive symptoms are contradictory. Several studies found no correlation between olfactory function and depressive symptoms ${ }^{\left({ }^{39}\right)}$ or no significant differences in olfactory test scores between depressed patients and controls ${ }^{(40)}$, whereas others show that odour identification and sensitivity are reduced in patients with symptoms of depression ${ }^{(12,41,42)}$. The current data indicate that depressive symptoms are associated with olfactory deficits in a population-based sample of older adults; however, our cross-sectional results do not clarify the directionality of the relationship.

Table 3. Determinants of gustatory and olfactory ID scores.

\begin{tabular}{lcc|cc}
\hline & Olfactory ID score & \multicolumn{2}{c}{ Gustatory ID score } \\
\hline Variable & Standardized Beta & p-value & Standardized Beta & p-value \\
\hline Sex & $\mathbf{0 . 1 0 0}$ & $<\mathbf{0 . 0 0 1}$ & $\mathbf{0 . 1 9 6}$ & $\mathbf{0 . 0 0 1}$ \\
Age & $\mathbf{- 0 . 2 2 4}$ & $<\mathbf{0 . 0 0 1}$ & $\mathbf{0 . 0 6 4}$ & -0.011 \\
BMI & 0.034 & 0.100 & -0.006 & 0.604 \\
Depressive symptoms (CES-D) & $\mathbf{- 0 . 0 7 4}$ & $<\mathbf{0 . 0 0 1}$ & 0.772 & $\mathbf{0 . 0 5 3}$ \\
Education & $\mathbf{0 . 1 2 1}$ & $<0.001$ & 0.037 & 0.096 \\
Number of medications & 0.026 & 0.220 & & $\mathbf{0 . 0 1 8}$ \\
\hline
\end{tabular}

ID = identification

Multiple linear regression analysis for olfactory and gustatory identification scores, including variables sex, age, BMI, depressive symptoms (CES-D), education, number of medications.

Bold indicates variables that are significant predictors for olfactory or gustatory identification scores. 
Interestingly, a positive correlation between olfactory function and BMI was found. These data reinforce the findings of Simchen and colleagues ${ }^{(43)}$, which document the connection between high odour identification scores and high BMI in elderly participants by demonstrating a similar connection between low odour identification scores and low BMI. Since smell is a principal component of the flavor percept when tasting food, olfactory function is critical for dietary selection ${ }^{(31)}$. By extension, age-related reductions in olfactory function are likely to affect food pleasure and food intake and may lead to nutritional deficiencies, weight loss, and lower BMI ${ }^{(44,45)}$, an effect sometimes referred to as 'the anorexia of aging' (5). Although we found no correlation between taste identification scores and BMI, it is possible that the high prevalence of significant gustatory dysfunction in older adults may also contribute to this life-threatening disorder where older individuals lose their appetite leading to a great reduction in their body weight. These data call for a more thorough and clinical investigation of olfactory and gustatory function in the elderly population. Because of the cross-sectional design of the present study, we cannot render a directional claim of the obtained correlations. It is possible that a variable such as overall health status has affected both measures (BMI and olfactory function) here. However, this is only the first wave of a prospective longitudinal study, so more data in order to clarify these correlations will be collected.

A correlation was found between number of medications taken and gustatory function, in that the higher the number of medications taken, the better one's sense of taste. Although this may seem counterintuitive at first, we hypothesize the possibility that subjects not taking medication may in fact have poorer health because they are not treating possible symptoms, potentially due to lack of healthcare coverage, which is subsequently reflected in lower gustatory performance.

One of the limitations of this study is that the correlations between the chemical senses and physical health, medication use, cognitive function, and emotional health, albeit significant, are weak, and only a small percentage of the variance is explained. Moreover, although both the five-item olfactory identification test ${ }^{(18)}$ and the gustatory identification test ${ }^{(17)}$ have both been demonstrated to have a good validity, the allowed variation is not large which might hamper their power. Future studies warrant more extensive chemosensory tests to evaluate these specific correlations in further detail.

In conclusion, this study estimated population prevalence of significant gustatory and olfactory dysfunction in communityresiding older adults to be $14.8 \%$ and $2.7 \%$, respectively, corresponding to nearly 11 million community residing older adults in the US, suffering from either olfactory or gustatory dysfunction. Moreover, the current data indicate that BMI and depressive symptoms are correlated with olfactory, but not gustatory, function and that age, sex, and education are significant predictors of odour and taste identification performance. More importantly, these findings demonstrate that even the use of brief tests can confirm and extend previous knowledge of correlations between the chemical senses and clinically relevant measures of health in a large community sample of older adults.

\section{ACKNOWLEDGEMENTS}

We would like to thank Dr. P. Schumm for his useful comments regarding the statistical analyses. This work was supported by the National Institutes of Health (grant number 5R01AG021487), including the National Institute on Aging; the Office on Research on Women's Health; and the Office of AIDS Research. Additional financial support was provided by the National Opinion Research Center.

\section{AUTHORSHIP CONTRIBUTION}

SB contributed to analysis and interpretation of data. STL, MKM, JNL contributed to concept and design of the study, and analysis and interpretation of the data. TH contributed to concept and design of the study. All authors contributed to preparation of the manuscript, and approved the final version of the manuscript.

\section{CONFLICT OF INTEREST}

None of the authors have any conflict of interest.

\section{REFERENCES}

1. Doty RL, Shaman P, Applebaum SL, Giberson R, Siksorski L, Rosenberg L. Smell identification ability: Changes with age. Science. 1984; 226: 1441-1443.

2. Congdon N, O'Colmain B, Klaver CC et al. Causes and prevalence of visual impairment among adults in the United States. Arch Ophthalmol. 2004; 122: 477-485.

3. Cruickshanks KJ, Wiley TL, Tweed TS et al. Prevalence of hearing loss in older adults in Beaver Dam, Wisconsin. The Epidemiology of Hearing Loss Study. Am J Epidemiol. 1998; 148: 879-886.

4. Schiffman SS. Taste and smell losses in normal aging and disease. JAMA. 1997; 278: 1357-1362.

5. Hays NP, Roberts SB. The anorexia of aging in humans. Physiol Behav. 2006; 88: 257-266.

6. Roessner V, Bleich S, Banaschewski T, Rothenberger A. Olfactory deficits in anorexia nervosa. Eur Arch Psychiatry Clin Neurosci. 2005; 255: 6-9.

7. Schreder T, Albrecht J, Kleemann AM et al. Olfactory performance of patients with anorexia nervosa and healthy subjects in hunger and satiety. Rhinology. 2008; 46: 175-183.

8. Boesveldt S, Verbaan D, Knol DL et al. A comparative study of odour identification and odour discrimination deficits in Parkinson's disease. Mov Disord. 2008; 23: 1984-1990.

9. Doty RL, Deems DA, Stellar S. Olfactory dysfunction in parkinsonism: A general deficit unrelated to neurologic signs, disease stage, or disease duration. Neurology. 1988; 38: 1237-1244.

10. Larsson M, Semb H, Winblad B, Amberla K, Wahlund LO, Backman L. Odour identification in normal aging and early Alzheimer's disease: effects of retrieval support. Neuropsychology. 1999; 13: 47-53.

11. Murphy C. Loss of olfactory function in dementing disease. Physiol Behav. 1999; 66: 177-182.

12. Pause BM, Miranda A, Goder R, Aldenhoff JB, Ferstl R. Reduced olfactory performance in patients with major depression. J Psychiatr Res. 2001; 35: 271-277.

13. Striebel KM, Beyerstein B, Remick RA, Kopala L, Honer WG. Olfactory identification and psychosis. Biol Psychiatry. 1999; 45: 1419-1425.

14. Murphy C, Schubert CR, Cruickshanks KJ, Klein BEK, Klein $\mathrm{R}$, Nondahl DAM. Prevalence of olfactory impairment in older 
adults. JAMA. - J Am Med Assoc. 2002; 288: 2307-2312.

15. Wysocki CJ, Gilbert AN. National Geographic Smell Survey. Effects of age are heterogenous. Ann N Y Acad Sci. 1989; 561: 12-28.

16. Vennemann MM, Hummel T, Berger K. The association between smoking and smell and taste impairment in the general population. J Neurol. 2008; 255: 1121-1126.

17. Mueller C, Kallert S, Renner B et al. Quantitative assessment of gustatory function in a clinical context using impregnated ,taste strips". Rhinology. 2003; 41: 2-6.

18. Mueller C, Renner B. A new procedure for the short screening of olfactory function using five items from the „Sniffin' Sticks“ identification test kit. Am J Rhinol. 2006; 20: 113-116.

19. Lindau ST, Schumm LP, Laumann EO, Levinson W, O'Muircheartaigh CA, Waite LJ. A study of sexuality and health among older adults in the United States. N Engl J Med. 2007; 357: $762-774$.

20. Schumm LP, Williams S, Leitsh S et al. Assessment of Sensory Function in the National Social Life, Health and Aging Project. J Gerontol. 2009.

21. Landis BN, Welge-Luessen A, Bramerson A et al. «Taste Strips» a rapid, lateralized, gustatory bedside identification test based on impregnated filter papers. J Neurol. 2009; 256: 242-248.

22. Bartlett SJ, Kolodner K, Butz AM, Eggleston P, Malveaux FJ, Rand CS. Maternal depressive symptoms and emergency department use among inner-city children with asthma. Arch Pediatr Adolesc Med. 2001; 155: 347-353.

23. Radloff LS. The CES-D scale: A self-report depression scale for research in the general population. Applied Psychological Measurement. 1977; 1: 385-401.

24. Brämerson A, Johansson L, Ek L, Nordin S, Bende M. Prevalence of olfactory dysfunction: The Skövde population-based study. Laryngoscope. 2004; 114: 733-737.

25. Landis BN, Konnerth CG, Hummel T. A study on the frequency of olfactory dysfunction. Laryngoscope. 2004; 114: 1764-1769.

26. Fusari A, Ballesteros S. Identification of odours of edible and nonedible stimuli as affected by age and gender. Behavioral Research Methods. 2008; 40: 752-759.

27. Ayabe-Kanamura S, Saito S, Distel H, Martinez-Gomez M, Hudson R. Differences and similarities in the perception of everyday odours: A Japanese-German cross-cultural study. Ann N Y Acad Sci. 1998; 855: 694-700.

28. Deems DA, Doty RL, Settle RG et al. Smell and taste disorders, a study of 750 patients from the University of Pennsylvania Smell and Taste Center. Acta Otolaryngol Head Neck Surg. 1991; 117: 519-528.

29. Pribitkin E, Rosenthal MD, Cowart BJ. Prevalence and causes of severe taste loss in a chemosensory clinic population. Ann Otol Rhinol Laryngol. 2003; 112: 971-978.

30. Cowart BJ. Relationships between taste and smell across the adult life span. Ann N Y Acad Sci. 1989; 561: 39-55.

31. Murphy C. The chemical senses and nutrition in older adults. J Nutr Elder. 2008; 27: 247-265.

32. Nordin S, Bramerson A, Bringlov E, Kobal G, Hummel T, Bende $\mathrm{M}$. Substance and tongue-region specific loss in basic taste-quality identification in elderly adults. Eur Arch Otorhinolaryngol. 2007; 264: 285-289.
33. Ahne G, Erras A, Hummel T, Kobal G. Assessment of gustatory function by means of tasting tablets. Laryngoscope 2000; 110 : 1396-1401.

34. Bartoshuk LM. Taste. Robust across the age span? Ann N Y Acad Sci 1989; 561: 65-75

35. Hummel T, Kobal G, Gudziol H, Mackay-Sim A. Normative data for the "Sniffin" Sticks" including tests of odour identification, odour discrimination, and olfactory thresholds: An upgrade based on a group of more than 3000 subjects. Eur Arch Otorhinolaryngol. 2007; 264: 237-243.

36. Seiberling KA, Conley DB. Aging and olfactory and taste function. Otolaryngol Clin North Am. 2004; 37: 1209-1228.

37. Fikentscher R, Roseburg B, Spinar H, Bruchmuller W. Loss of taste in the elderly: sex differences. Clin Otolaryngol Allied Sci. 1977; 2: 183-189.

38. Larsson M. Odour memory: A memory systems approach. In Rouby C, Schaal B, Dubois D, Gervais R, Holley A, eds. Olfaction, taste, and cognition. Cambridge: Cambridge University Press, UK. 2002; 231-245.

39. Scinska A, Wrobel E, Korkosz A et al. Depressive symptoms and olfactory function in older adults. Psychiatry Clin Neurosci. 2008; 62: $450-456$

40. Amsterdam JD, Settle RG, Doty RL, Abelman E, Winokur A. Taste and smell perception in depression. Biol Psychiatry. 1987; 22: 1481-1485.

41. Lombion-Pouthier S, Vandel P, Nezelof S, Haffen E, Millot JL. Odour perception in patients with mood disorders. J Affect Disord. 2006; 90: 187-191.

42. Pollatos O, Albrecht J, Kopietz R et al. Reduced olfactory sensitivity in subjects with depressive symptoms. J Affect Disord. 2007; 102: 101-108.

43. Simchen U, Koebnick C, Hoyer S, Issanchou S, Zunft H-JF. Odour and taste sensitivity is associated with body weight and extent of misreporting of body weight. European Journal of Clinical Nutrition. 2006; 60: 698-705.

44. Duffy VB, Backstrand JR, Ferris AM. Olfactory dysfunction and related nutritional risk in free-living, elderly women. J Am Diet Assoc. 1995; 95: 879-884.

45. Schiffman SS, Graham BG. Taste and smell perception affect appetite and immunity in the elderly. Eur J Clin Nutr. 2000; 54 Suppl 3: S54-S63.

Sanne Boesveldt

Division of Human Nutrition

Wageningen University

PO Box 8129

6700 EV Wageningen

the Netherlands

Tel: +31-(0)317-482 111

E-mail: sanne.boesveldt@wur.nl 